\title{
Anti-Ma2-Associated Limbic Encephalitis after Termination of Immune Checkpoint Inhibitor Therapy for Malignant Pleural Mesothelioma
}

\author{
Rei Hashimoto Etsuko Tanabe Yoshihisa Otsuka Yukihiro Yoneda \\ Yasufumi Kageyama \\ Division of Neurology, Hyogo Prefectural Amagasaki General Medical Center, Amagasaki, \\ Japan
}

Keywords

Anti-Ma2 antibody · Encephalitis · Immune checkpoint inhibitor · Paraneoplastic neurological syndrome $\cdot$ Mesothelioma

\section{Abstract}

Neurological adverse events of immune checkpoint inhibitor (ICI) therapy mostly develop within 3 months after initiation of $\mathrm{ICl}$ treatment. An 82-year-old male with malignant pleural mesothelioma developed anti-Ma2-associated limbic encephalitis at a delay of 18 months after the start of nivolumab therapy (3 months after termination of a 15-month course of ICl treatment). Immunotherapy with steroids and immunoglobulins resulted in moderate neurological improvement. Over the next year, malignant pleural mesothelioma gradually worsened, while the anti-Ma2 antibody test remained positive. Anti-Ma2 paraneoplastic encephalitis may occur after a delay following the discontinuation of $\mathrm{ICl}$ therapy.

\section{Introduction}

The neurological adverse events of immune checkpoint inhibitors (ICIs) mostly develop within the first 3 months after the initiation of ICI treatment, with a median delay of 2 months [1]. Autoimmune anti-Ma2 paraneoplastic neurological syndromes generally occur in patients with 
testicular tumors (40\%) and rarely in those with malignant pleural mesothelioma (5\%) [2]. Incidence of anti-Ma2 paraneoplastic encephalitis has rapidly increased after the introduction of ICI therapy [1, 3-7]. We report a case of anti-Ma2 limbic encephalitis developing 3 months after the termination of a 15-month course of nivolumab therapy for malignant pleural mesothelioma.

\section{Case Report/Case Presentation}

An 82-year-old male with biopsy-confirmed stage I malignant pleural mesothelioma underwent chemotherapy after pleurodesis treatment. After a 4-month course of cytotoxic chemotherapy with carboplatin and pemetrexed as first-line therapy, he received 31 courses of anti-programmed death-1 (PD-1) receptor monoclonal antibody nivolumab (240 mg/body) for 15 months as a second-line therapy. However, his malignant pleural mesothelioma was refractory with enlarged right axillary lymph nodes, and the chemotherapy regimen was switched to 3 courses of gemcitabine plus vinorelbine as third-line therapy. Eighteen months after the initial dose of nivolumab ( 3 months after the last dose), he presented with partial seizures of the right face, followed by memory deficits and speech difficulties.

Upon presentation to the neurology clinic, the patient exhibited somnolence and cognitive impairment, as demonstrated by a Mini-Mental State Examination (MMSE) score of 14/30 points. On brain magnetic resonance imaging (MRI) and fluid-attenuated inversion recovery images demonstrated high-signal lesions in the right mesial temporal and bilateral basal frontal regions (Fig. 1). Diffusion-weighted images showed high signal lesions in the left basal frontal area without gadolinium enhancement on T1 images. Brain single-photon emission computed tomography with 99mTc showed decreased blood flow in the right mesial temporal and basal frontal areas. Upon analysis, the cerebrospinal fluid (CSF) was acellular with a mildly high protein level $(63.3 \mathrm{mg} / \mathrm{dL})$, and oligoclonal IgG bands. Neither the herpes simplex virus nor the varicella-zoster virus was detected in the CSF by the polymerase chain reaction test. The serum anti-Ma2 antibody was positive, but other onconeural antibodies (anti-amphiphysin, CV2, - $\mathrm{Ri},-\mathrm{Yo},-\mathrm{Hu}$, -recoverin, -SOX1, -titin, -zic4, -GAD65, and -Tr), anti-NMDA receptor antibody, anti-LGI-1 antibody, antiCASPR2 antibody, anti-AQP-4 antibody, and anti-MOG antibody were all negative. The CSF was not screened for anti-Ma2 antibodies. Although anti-Ma2 antibody is often associated with testicular tumors, a subsequent testicular ultrasound and tumor marker test results were negative.

We considered a diagnosis of anti-Ma2 limbic encephalitis related to nivolumab and he underwent 2 sessions of intravenous high-dose methylprednisolone therapy $(1,000 \mathrm{mg} / \mathrm{body}$, 3 days). His disorientation and memory subsequently improved - as demonstrated by an improved MMSE score of 24/30 points - and his speech spontaneity increased. MRI showed mild improvement in the left frontal lesion. Although he was additionally treated with intravenous immunoglobulin $(0.4 \mathrm{~g} / \mathrm{kg}, 5$ days), no further improvement was observed. Levetiracetam was administered to treat the patient's seizures, and there was no recurrence until he was discharged on hospital day 36.

We then restarted third-line chemotherapy with gemcitabine and vinorelbine, repeated this combination for 6 courses over 9 months, and then discontinued chemotherapy secondary to right hilar lymph node enlargement. Two months later, secondary seizures occurred and he was re-hospitalized. Brain MRI showed atrophy in the right medial temporal region and mild enlargement of the high signal lesions in the basal frontal regions (Fig. 1). CSF examination demonstrated no elevated cell counts and negative oligoclonal bands, but the serum anti-Ma2 antibody remained positive. The chest computed tomography exhibited marked enlargement of the right hilar lymph nodes with no further pleural thickening (Fig. 1). After 1 session of intravenous methylprednisolone therapy, there was no recurrence of seizures, and he was discharged with a modified Rankin Scale score of 3.

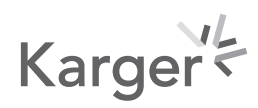




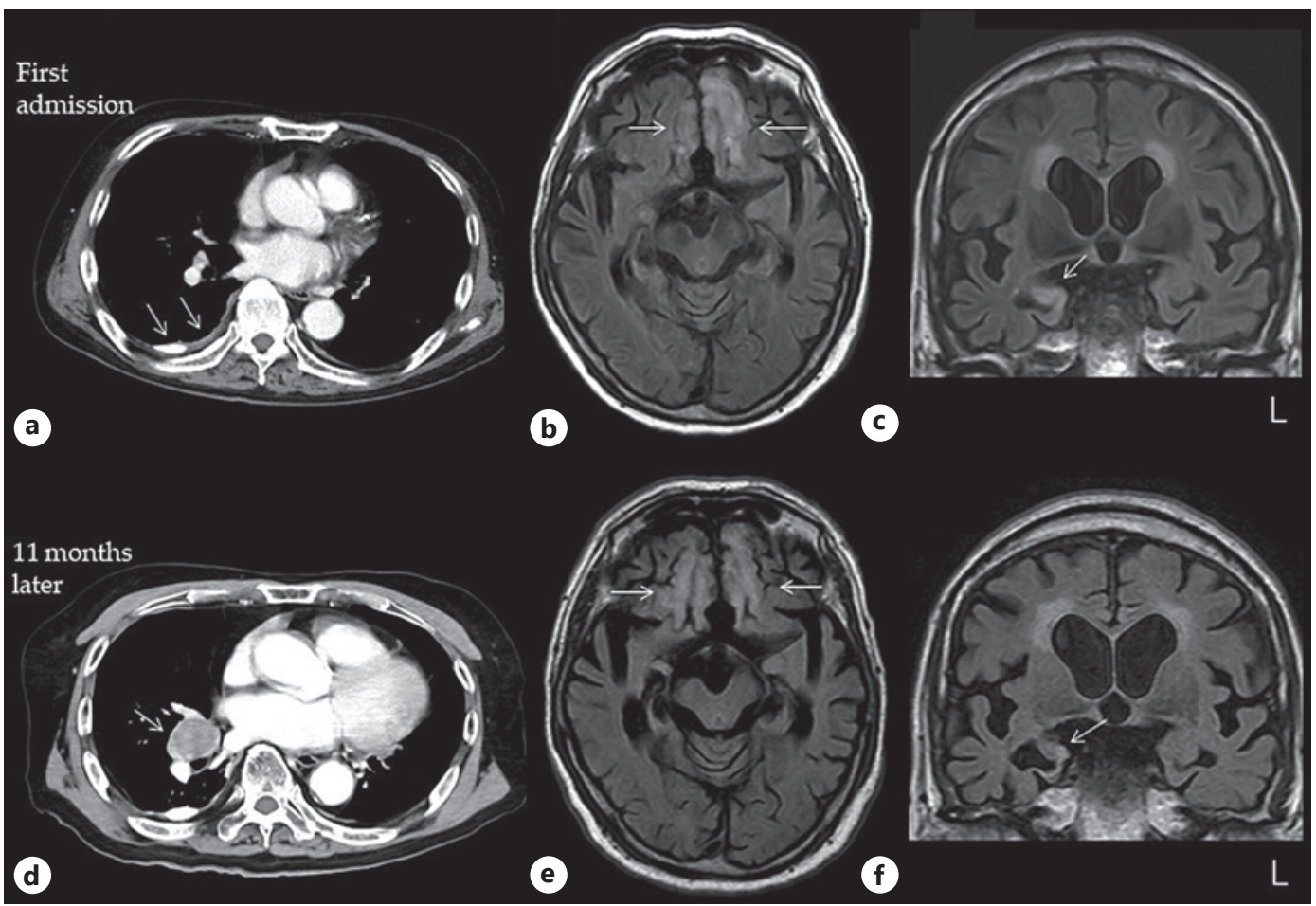

Fig. 1. a Chest CT showing pleural thickening of the lower right lung lobe with calcified lesions. b, c Fluidattenuated inversion recovery images on brain MRI demonstrating high-signal lesions in the right mesial temporal and bilateral basal frontal regions. d Chest CT exhibiting an enlargement of the right hilar lymph nodes. e, $\mathbf{f}$ Brain MRI showing atrophy in the right medial temporal region and mild enlargement of the highsignal lesions in the basal frontal regions. CT, computed tomography; MRI, magnetic resonance imaging.

\section{Discussion}

This patient developed anti-Ma2 paraneoplastic limbic encephalitis after a delay of 18 months following the initiation of nivolumab therapy ( 3 months after the termination of a 15-month course of nivolumab therapy) for malignant pleural mesothelioma. In previous reports [3, 7], 2 patients with malignant pleural mesothelioma developed anti-Ma2 encephalitis during ICI treatment. In patients with various active malignancies, the median interval between the onset of encephalitis and the start of ICI treatment was 4 months (range, 2-8 months) [3]. Uncommonly, our patient developed anti-Ma2 encephalitis during the next-step chemotherapy, with a delay of 3 months after the termination of a 15-month course of nivolumab therapy. Therefore, we could not scientifically confirm whether the ICI therapy was directly linked to the development of anti-Ma2 encephalitis. Nivolumab has a long-lasting anticancer effect after administration, with the half-life of a single dose of nivolumab being 12-20 days [8]. The PD-1 receptor occupancy in the Tlymphocytes in the blood is dose-independent; the washout speed is very slow, remaining above $70 \%$ for $>2$ months and decreasing gradually after 85 days [8]. In our patient, although limbic encephalitis occurred 3 months after the last dose of nivolumab, the blood concentrations of nivolumab and PD-1 receptor occupancy were probably maintained. Recently, a new oncological concept called "delayed immune-related events" that occur $>3$ months after ICI therapy termination has been proposed [9]. In our patient, overlap of prolonged nivolumab treatment and refractory malignant pleural mesothelioma may have had a cumulative effect on the development of delayedonset anti-Ma2 encephalitis. 
Interestingly, in the 2 previously reported patients with anti-Ma2 encephalitis and malignant pleural mesothelioma who underwent ICI treatments [3, 7], frozen blood samples obtained before the ICI therapy showed the presence of the anti-Ma2 antibody. These findings suggest that these patients were neurologically asymptomatic anti-Ma2 antibody carriers. In our case, pre-ICI therapy blood samples were unavailable.

\section{Conclusion}

Autoimmune anti-Ma2 paraneoplastic encephalitis may occur even after the termination of ICI treatment.

\section{Acknowledgments}

We would like to thank Dr. Masatoshi Shimada, M.D (respiratory medicine), for the clinical care of this patient. The Editage (www.editage.com) supported an English language editing.

\section{Statement of Ethics}

A written informed consent for publication of data and images was obtained from the patient and his family. This study was conducted ethically in accordance with the World Medical Association Declaration of Helsinki.

\section{Conflict of Interest Statement}

The authors have no conflicts of interest to declare.

\section{Funding Sources}

No funding was received for the publication of this case report.

\section{Author Contributions}

All authors read and approved the final version of manuscript. R.H. examined the patient and contributed to data analysis and drafting of the manuscript. E.T. examined the patient, contributed to data analysis, and made a preliminary presentation at the local conference. Y.Y. examined the patient and contributed to data analysis and revision of the manuscript. Y.O. and Y.K. contributed to data analysis and revision of the manuscript.

\section{Data Availability Statement}

All data generated or analyzed during this study are included in this article. Further inquiries can be directed to the corresponding author. 


\section{References}

1 Sechi E, Markovic SN, McKeon A, Dubey D, Liewluck T, Lennon VA, et al. Neurologic autoimmunity and immune checkpoint inhibitors: Autoantibody profiles and outcomes. Neurology. 2020 0ct;95(17):e2442-52.

2 Ortega Suero G, Sola-Valls N, Escudero D, Saiz A, Graus F. Anti-Ma and anti-Ma2-associated paraneoplastic neurological syndromes. Neurologia. 2018 Jan-Feb;33(1):18-27.

3 Vogrig A, Fouret M, Joubert B, Picard G, Rogemond V, Pinto AL, et al. Increased frequency of anti-Ma2 encephalitis associated with immune checkpoint inhibitors. Neurol Neuroimmunol Neuroinflamm. 2019 Aug;6(6):e604.

4 Vogrig A, Muñiz-Castrillo S, Joubert B, Picard G, Rogemond V, Marchal C, et al. Central nervous system complications associated with immune checkpoint inhibitors. J Neurol Neurosurg Psychiatry. 2020 Jun;91(7):772-8.

5 Marini A, Bernardini A, Gigli GL, Valente M, Muñiz-Castrillo S, Honnorat J, et al. Neurologic adverse events of immune checkpoint inhibitors: a systematic review. Neurology. 2021 Apr;96(16):754-66.

6 Zivelonghi C, Zekeridou A. Neurological complications of immune checkpoint inhibitor cancer immunotherapy. J Neurol Sci. 2021 May;424:117424.

7 Shibaki R, Murakami S, Oki K, Ohe Y. Nivolumab-induced autoimmune encephalitis in an anti-neuronal autoantibody-positive patient. Jpn J Clin Oncol. 2019 Aug;49(8):793-4.

8 Brahmer JR, Drake CG, Wollner I, Powderly JD, Picus J, Sharfman WH, et al. Phase I study of single-agent antiprogrammed death-1 (MDX-1106) in refractory solid tumors: safety, clinical activity, pharmacodynamics, and immunologic correlates. J Clin Oncol. 2010 Jul;28(19):3167-75.

9 Couey MA, Bell RB, Patel AA, Romba MC, Crittenden MR, Curti BD, et al. Delayed immune-related events (DIRE) after discontinuation of immunotherapy: diagnostic hazard of autoimmunity at a distance. J Immunother Cancer. 2019 Jul;7(1):165. 